\title{
Aberrant expression of long non-coding RNAs (IncRNAs) is involved in brain glioma development
}

Yi Ding ${ }^{1}$, Xinfa Wang ${ }^{1}$, Junchen Pan², Minjun Ji ${ }^{3}$, Zhengxiang Luo ${ }^{4}$, Penglai Zhao ${ }^{4}$, Yansong Zhang ${ }^{4}$, Gang Wang ${ }^{1}$

${ }^{1}$ Department of Neurosurgery, Children's Hospital of Nanjing Medical University, Nanjing, China

2Department of Neurosurgery, Nanjing BenQ hospital, Nanjing, China

${ }^{3}$ Department of Neurosurgery, Nanjing Medical University, Nanjing, China

${ }^{4}$ Department of Neurosurgery, Nanjing Brian Hospital Affiliated to Nanjing Medical

University, Nanjing, China

Submitted: 27 March 2017

Accepted: 1 August 2017

Arch Med Sci 2020; 16 (1): 177-188

DOI: https://doi.org/10.5114/aoms.2020.91290

Copyright $\odot 2019$ Termedia \& Banach

\section{Abstract}

Introduction: Aberrant expression of long non-coding RNAs (IncRNAs) has been implicated in various diseases, including cancer. However, little is known about IncRNAs in human brain gliomas.

Material and methods: We examined IncRNA profiles from three glioma specimens using IncRNA expression profiling microarrays. Quantitative real-time RT-PCR was used to analyze the differential expression of raw intensities of IncRNA expression in glioma and peritumoral tissues.

Results: We found 4858 IncRNAs to be differentially expressed between tumor tissue and peritumoral tissue. Of these, 2845 IncRNAs were up-regulated (fold change > 3.0) and 2013 were down-regulated (fold change $<1 / 3$ ). A total of 4084 messenger RNAs were also differentially expressed, including 2280 up-regulated transcripts (fold change > 3.0) and 1804 that were down-regulated (fold change $<1 / 3$ ). Consistent with the microarray data, qPCR confirmed differential expression of these 6 IncRNAs (ak125809, ak098473, uc002ehu.1, bc043564, NR_027322, and uc003qmb.2) between tumor and peritumoral tissue. We next established co-expression networks of differentially expressed IncRNAs and mRNAs. Many mRNAs, such as LOC729991, NUDCD1, SHC3, PDGFA, and MDM2, and InCRNAs, such as ENST00000425922, ENST00000455568, uc002ukz.1, ENST00000502715, and NR_027873, have been shown to play important roles in glioma development. Consistent with this, pathway analysis revealed that "GLIOMA" (KEGG Pathway ID: hsa05214) was significantly enriched in tumor tissue. Conclusions: Our data suggest that altered expression of IncRNAs may be a critical determinant of tumorigenesis in glioma patients.

Key words: IncRNAs, glioma, microarrays.

\author{
Corresponding authors: \\ Yansong Zhang \\ Department \\ of Neurosurgery \\ Nanjing Brian Hospital \\ Affiliated to Nanjing \\ Medical University \\ 025 Nanjing, China \\ Phone: +862586862793 \\ E-mail: yan_song_ \\ zhang@126.com \\ Gang Wang \\ Department \\ of Neurosurgery \\ Nanjing Children's Hospital \\ Nanjing, China \\ E-mail: wang509111@163.com
}

\section{Introduction}

Gliomas are the most common and most severe form of primary brain cancer, accounting for approximately $52 \%$ of all primary brain tumors and $20 \%$ of intracranial tumors [1]. Gliomas arise from malignant transformation of glial cells and can be subdivided into astrocytomas, oligodendrogliomas, ependymomas, and mixed gliomas according to their clinical and pathological features. Malignant glioma ranks second (behind only stroke) among all nerve-associated diseases in mortality rate [1]. 
Even with current surgical and chemotherapy options, the median survival time of patients with malignant glioma or glioblastoma is only 14 months [2]. While great strides in glioma research have been made over the last two decades, the exact pathological mechanism underlying the disease has not been defined [3]. Thus, additional research is required to not only better understand the disease itself but also to improve prognosis and therapeutic options for patients.

Long non-coding RNAs (IncRNAs) are regulatory RNA transcripts typically over 200 nucleotides in length [4]. In mammals, IncRNAs are 6-8 times more abundant than mRNAs and are encoded by 4-9\% of the entire genome [5]. While originally considered the "noise" of transcription, IncRNAs are now known to be vital in multiple molecular processes, including chromatin modification $[5,6]$, transcriptional activation [7], and post-transcriptional regulation [8]. LncRNAs have also been shown to be critical in a number of physiological and pathological processes [9-13].

Aberrant expression of IncRNAs has been linked to tumorigenesis $[14,15]$. For example, one study used a hepatocellular carcinoma-specific cDNA microarray to identify increased expression of the InCRNA HULC in tumor tissue [15]. Additionally, InCRNA-HULC was also detectable in the plasma of hepatocellular carcinoma patients, thus making it a potential diagnostic biomarker [15]. LnCRNA-HULC (hepatocellular carcinoma up-regulated long non-coding RNA) was also shown to regulate the expression of multiple genes in hepatocellular carcinoma cells, strengthening the link between this IncRNA and tumorigenesis [12]. Overexpression of the IncRNA maternally expressed gene 3 (MEG3) inhibited proliferation and induced apoptosis of both U87 MG and U251 human glioma cell lines [16]. The authors showed that this effect was due to the interaction among MEG3, p53, MDM2, and GDF15 [17]. Another study examined the expression profiles of both IncRNAs and mRNAs in glioblastoma; the authors found two IncRNAs, ASLNC22381 and ASLNC2081, that were related to the disease via their regulation of insulin-like growth factor 1 (IGF-1) [18]. Zhang et al. used a combination of publicly available data and their own microarray analysis to assess IncRNA expression in astrocytic and oligodendroglial glioma [19]. Interestingly, they found a significant correlation between the expression of specific IncRNAs and both malignancy grade and histological differentiation [19]. The follow-up to these findings has been limited due to insufficient quantity of samples and to limited IncRNAs included on microarray platforms. Despite this, much of this earlier work laid the foundation for a role for IncRNAs in glioma development - a topic that deserves more extensive study.
Here, we used IncRNA expression profiling microarray to identify differentially expressed IncRNAs in clinical glioma samples compared to normal tissue. Six IncRNAs (ak125809, ak098473, uc002ehu.1, bc043564, NR_027322, and uc$003 q \mathrm{mb}$.2) were selected and validated by quantitative real-time RT-PCR (qPCR). We established an mRNA/IncRNA co-expression network to identify key regulators of glioma development. The data we present here may provide a better understanding of the disease and new therapeutic strategies to combat it in patients.

\section{Material and methods}

\section{Sample collection}

Forty cases of glioma and peritumoral tissue were obtained from the Neurosurgery Department of Nanjing Brain Hospital Affiliated with Nanjing Medical University. Peritumoral tissue was obtained from both a surgical approach and peripheral edema tissue. Pathological categories were evaluated by the hospital's pathologist (ID: NJNKYY2012LS027). Written informed consent was obtained from all patients. This study was approved by the Ethics Committee of the University. Following collection, specimens were shipped and preserved in liquid nitrogen. Details are shown in supplement material 1. The protocol for the tissue collection was as follows: (1) Record the information of tumor tissue on the tissue cryopreserved tube; (2) wash the tumor tissue with saline, use ophthalmic scissors, ophthalmic tweezers to remove blood vessels, envelopes and necrotic tissue; (3) cut the tumor tissue into $2 \mathrm{~mm}$ thick slices; (4) flatten tissue on sterile gauze, and remove the surface liquid of tissue sections; (5) quickly immerse the tissue in liquid nitrogen, not less than 5 minutes; (6) quickly move the tissue into the tissue cryopreservation tube, tighten the tube and move into the liquid nitrogen tank for preservation.

\section{RNA extraction}

Total RNA was isolated with TRIzol reagent (Invitrogen, CA, USA) following the single-step method [20]: 1) Glioma and adjacent tissues were pulverized with a mortar and pestle in liquid nitrogen. Tissue samples were homogenized in $1 \mathrm{ml}$ of Trizol per $50 \mathrm{mg}$ of tissue until liquid nitrogen evaporated. 2) The homogenate was pipetted up and down several times and transferred to an Eppendorf tube. 3) Homogenized samples were incubated for $5 \mathrm{~min}$ at room temperature and then centrifuged at $12,000 \mathrm{rpm}$ for $5 \mathrm{~min}$ according to the protocol. Next, $200 \mu \mathrm{l}$ of chloroform was added per $1 \mathrm{ml}$ of TRIzol. Samples were incubated for $15 \mathrm{~min}$ at room temperature and centrifuged at $12,000 \mathrm{rpm}$ for 
$15 \mathrm{~min}$ at $4^{\circ} \mathrm{C}$. The upper soluble layer was isolated and mixed with $0.5 \mathrm{ml}$ of isopropanol per $1 \mathrm{ml}$ of TRIzol. Samples were incubated for $10 \mathrm{~min}$ at room temperature and centrifuged at $12,000 \mathrm{rpm}$ for $10 \mathrm{~min}$ at $4^{\circ} \mathrm{C}$. The supernatant was removed and washed with $1 \mathrm{ml}$ of $75 \%$ ethanol. Samples were then centrifuged at $8,000 \mathrm{rpm}$ for $5 \mathrm{~min}$ at $4^{\circ} \mathrm{C}$. This was followed by a brief incubation at room temperature to allow the samples to dry. Finally, RNA samples were dissolved in $50 \mu$ l of DEPC water and stored at $-80^{\circ} \mathrm{C}$ until use. The concentration of each sample was determined using the NanoDrop ND-1000 Spectrophotometer (NanoDrop Technologies, Inc., USA). RNA integrity was evaluated by electrophoresis on a denaturing agarose gel.

\section{LncRNA and mRNA microarrays}

Total RNA from both glioma (0301, 0605, and 114982) and peritumoral tissue (0601) was isolated and converted into double-stranded complementary DNA (cDNA). The synthesized cDNAs were labeled and hybridized to Arraystar Human IncRNA Microarray V2.0 (Arraystar, Rockville, MD) containing probes for 33,045 IncRNAs and 30,215 mRNAs identified from both publications and authoritative databases, such as RefSeq, UCSC Knowngenes, and Ensembl. Each transcript was represented by a specific exon or splice junction probe, which could accurately identify individual transcripts. Positive control probes and negative control probes for housekeeping genes were also printed onto the array [21].

\section{Microarray hybridization and data analysis}

Arraystar IncRNA Array Protocol: step 1 - Prepare the RNA sample using the following reagents and kits: TRIzol Reagent (Invitrogen Life Technologies), Biopulverizer (Biospec), and Mini-Bead-Beater-16 (Biospec); step 2 - Total RNA clean-up and quality control using the following reagents and instruments: RNeasy Mini Kit (Qiagen, p/n 74104), NanoDrop ND-1000, and Baseline-ZERO DNase (EPICENTRE, Cat. Nos. DB0711K); step 3 - Prepare labeling reaction with the following: Quick Amp Labeling Kit and One-Color (Agilent p/n 5190-0442); step 4 - Purify the labeled RNA using the RNeasy Mini Kit (Qiagen, p/n 74104) and NanoDrop ND1000; step 5 - Hybridization using the Agilent Gene Expression Hybridization Kit (Agilent, p/n 51885242), stainless hybridization chamber (Agilent, p/n G2534A), Hybridization Chamber gasket slides (Agilent, p/n G2534-60003), Hybridization oven (Agilent, p/n G2545A), and Hybridization oven rotator for Agilent Microarray Hybridization Chambers (Agilent, p/n G2530-60029); step 6 - Microarray Wash using Gene Expression Wash Buffer 1 (Agilent, $\mathrm{p} / \mathrm{n}$ 5188-5325), Gene Expression Wash Buffer 2 (Agilent, p/n 5188-5326), Magnetic stir bar (Corning, $p / n$ 401435), Magnetic stir plate (Corning, $p / n$ 6795-410), and Slide-staining dish with slide rack (Thermo Shandon, p/n 121); step 7 - Scanning performed with Agilent Microarray Scanner (Agilent p/n G2565BA); step 8 - Extract data using Agilent Feature Extraction software. Following scanning on the Agilent Scanner G2505B, acquired images were analyzed with Agilent Feature Extraction software (version 10.7.3.1). Data analysis and normalization were performed using the GeneSpring GX v11.5.1 software package (Agilent Technologies). All microarray work and data processing were carried out by KangChen Bio-tech (Shanghai, P.R. China) [22].

\section{Real-time quantitative polymerase chain reaction (RT-qPCR)}

Total RNA was extracted using TRIzol (Invitrogen, CA, USA). Reverse transcription was performed using the PrimeScript RT reagent Kit with gDNA Eraser (Perfect Real Time) (TaKaRa, Dalian, China) according to the manufacturer's protocol. Expression of IncRNAs (ak125809, ak098473, uc002ehu.1, bc043564, NR_027322, and uc003qmb.2) was determined using the SYBRGreen detection method (TaKaRa, Dalian, China). Samples were run on an ABI7500 System (Applied Biosystems, Foster City, CA). Primer sequences for IncRNA detection are listed in Table I. $\beta$-actin was

Table I. Primers for qPCR

\begin{tabular}{|c|c|c|}
\hline IncRNA & Forward 5'-3' & Reverse $5^{\prime}-3^{\prime}$ \\
\hline ak125809 & GGGAGGTTTTGCTACTTCTGTG & TGGTTCTTTGGGAGTGTTTGTT \\
\hline ak098473 & GGAATGTCTCCСCTCACСTC & AGCTTGATCCCTCGTCCTTTG \\
\hline uc002ehu.1 & TGTAGGATGCCACTGGAAATG & СTTCTGCGTGACAACTGAGGAT \\
\hline bc043564 & CTTTTTCGATCGGAACCGCC & GAGGACGCTCTTCСTCСTTG \\
\hline NR_027322 & ACCTACCCCGTACTCATCCC & TGGTGTTCTCGGCTTCTGTC \\
\hline uc003qmb.2 & TGTGGTATTTGTTCTCTTGGATTG & ACTTCGGAGGCAGTAGTAAAATCA \\
\hline$\beta$-actin & ТССТСТСССАAGTССАСАСA & GCACGAAGGCTCATCATTCA \\
\hline
\end{tabular}


used as a normalization control. The GPCR reaction was carried out in a total volume of $20 \mu \mathrm{l}$. The reaction consisted of $10 \mu \mathrm{l}$ of $2 \times$ SYBR Green Master mix, $1 \mu \mathrm{l}$ of PCR Forward Primer $(5 \mu \mathrm{mol} / \mu \mathrm{l})$, $1 \mu \mathrm{l}$ of PCR Reverse Primer $(5 \mu \mathrm{mol} / \mu \mathrm{l}), 1 \mu \mathrm{l}$ cDNA (10 ng), and $7 \mu$ of RNase-free water. The RT-qPCR reaction was performed as follows: initial denaturation at $95^{\circ} \mathrm{C}$ for $10 \mathrm{~min}, 40$ cycles of denaturation at $95^{\circ} \mathrm{C}$ for $15 \mathrm{~s}$, annealing at $60^{\circ} \mathrm{C}$ for $30 \mathrm{~s}$, and extension at $72^{\circ} \mathrm{C}$ for $30 \mathrm{~s}$, and a final extension at $72^{\circ} \mathrm{C}$ for $5 \mathrm{~min}$. Each sample was analyzed in triplicate. The threshold cycle (CT) value was calculated by the mean value. Quantitative differential expression was calculated according to the $2^{-\Delta \Delta c t}$ method [23]. Statistical analysis was conducted using Student's $t$ test and SPSS software (Version 16.0 SPSS Inc.). Statistical significance was set at $p<0.05$.

\section{Pathway analysis}

Differentially expressed mRNAs were examined by pathway analysis using the latest KEGG (Kyoto Encyclopedia of Genes and Genomes) database. A number of specific biological pathways were identified by enrichment of the differentially expressed mRNAs. For statistical significance, the $p$-value cut-off was set at 0.05 .

\section{Establishing co-expression networks}

Co-expression networks of IncRNAs and mRNAs were constructed by differential gene expression. Different networks were drawn based on calculations of different types of expression data. For example, the co-expression network in the control group was calculated by differential gene expression in control samples, while that in the experimental group was calculated by differential gene expression in experimental samples. We calculated the Pearson correlation coefficient for each pair of genes. Regulatory relationships were identified when the Pearson coefficients achieved statistical significance; subsequently, significant correlation pairs were chosen to construct a network. An array $M$ was constructed based on the regulatory relationship between genes and mapped into the network diagram. Within the network diagram, each node represents a single gene, and the edges between two nodes represent an interaction between genes, i.e. the correlation between IncRNA-mRNA, IncRNA-IncRNA, and mRNA-mRNA.

The degree of connectivity was used to assess the importance of the gene in the network. Degrees were used to quantify interactions between target genes. For any given gene, the higher its degree, the more regulatory relationships it is involved in with other genes in the network. Thus, the higher the degree, the more central the gene is in the network. Clustering coefficients were used to calculate the density of interactions among neighboring genes. The higher the clustering coefficient, the greater was the possibility that the gene was located in a core region and that neighboring genes were part of the core network as well. Core genes, which had high degree values, were defined as genes having extensive connections to other genes in the network. Comparisons between different networks were used to discover different gene connections in different networks.

\section{Statistical analysis}

Each experiment was performed in triplicate, and all data were represented as average values. Differences between average values were analyzed using Student's $t$ test. All statistical analyses were performed with SPSS software (Version 13.0; SPSS, Inc., Chicago, IL, USA).

Important biological pathways were identified with Fisher's test and the $\chi^{2}$ test. Both $p$-value and FDR (the $p$-value cut-off is 0.05) were used as selection criteria. Enrichment was calculated based on the following formula: $\operatorname{Re}=(\mathrm{nf} / \mathrm{n}) /(\mathrm{Nf} / \mathrm{N})(\mathrm{Re}=$ ENRICHMENT), where $n f$ is the number of differentially expressed genes in a particular gene catego$r y, n$ is the total number of genes in that category, $N f$ is the number of differentially expressed genes in the entire microarray, and $N$ is the total number of genes included in the microarray [24-26].

\section{Results}

\section{Aberrantly expressed IncRNAs in glioma}

We performed microarray analyses to identify IncRNAs and mRNAs that were differentially expressed between glioma tumor tissue (0301, 0605, and 114982) and peritumoral tissue (0601) (Figure $1 \mathrm{~A})$. Differences in expression are represented as fold change of tumor/normal (T/N). A fold change $\geq 3$ was used to filter the data (a negative fold change value indicated down-regulation and a positive value indicated up-regulation). In some cases, log2 fold changes are listed. $P$-value and fold change were both calculated by normalized expression data. Using these parameters, we identified thousands of IncRNAs that were differentially expressed between glioma and normal tissue. Importantly, these IncRNAs have been described in several publications and are also included in a number of databases, including UCSC knowngene, Ensembl, RefSeq NR, H-invDB, Fantom, Fantom stringent, RNAdb, NRED, misc_IncRNA, and UCR.

We next compared IncRNA expression between tumor tissue and matched peritumoral tissue. While more than 23,000 IncRNAs were detected in all tissues, only a few thousand were found to be significantly differentially expressed. An average 
A

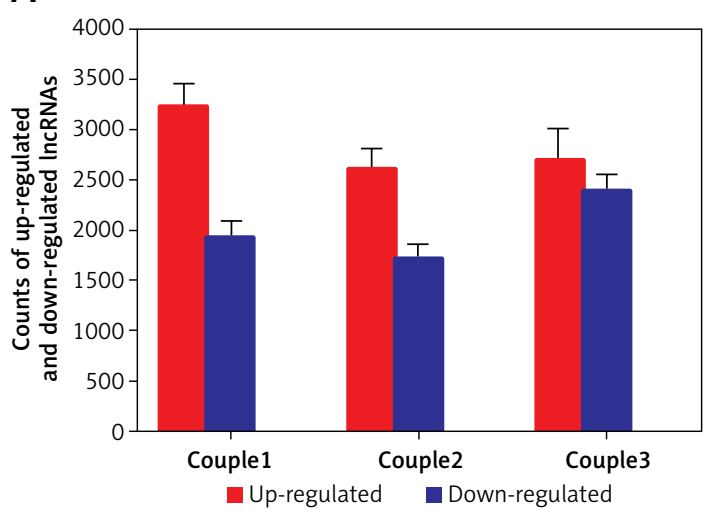

C

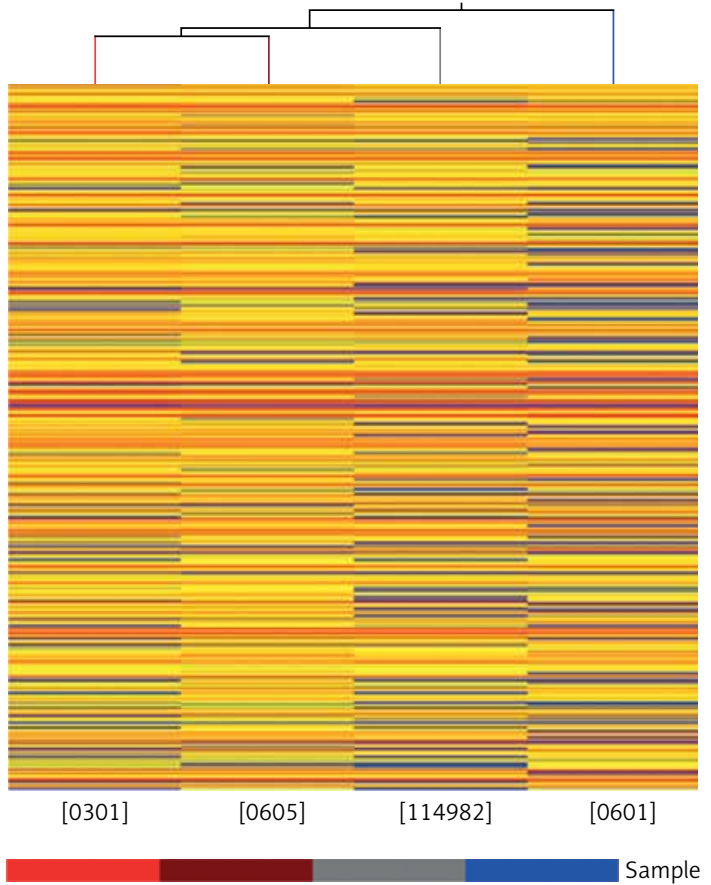

B

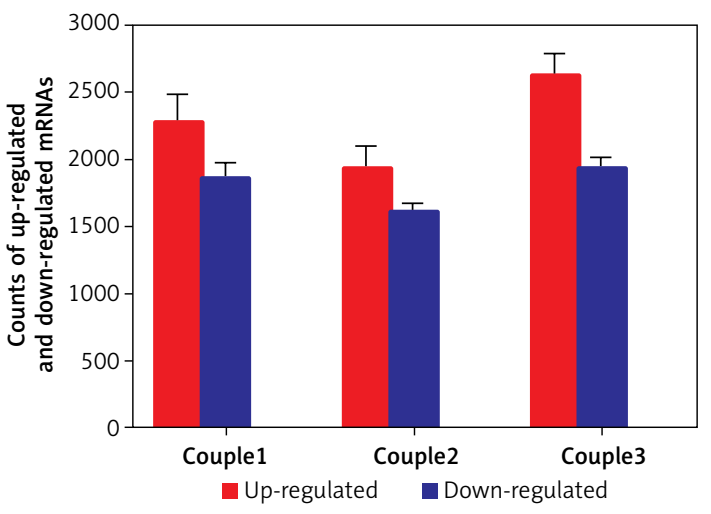

D

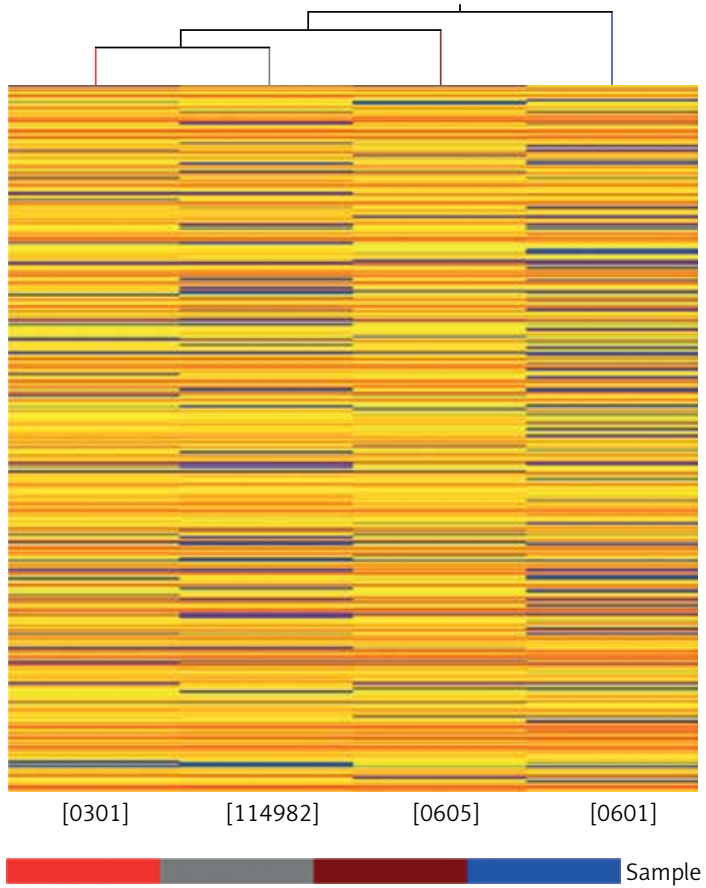

Figure 1. Microarray analysis was applied to detect the IncRNAs and mRNAs in glioma compared to normal peritumoral tissue. A - Differentially expressed IncRNAs were detected in gliomas. A, B - Differentially expressed mRNAs were detected in gliomas. C - Clustering data of IncRNAs in gliomas were analyzed. D - Clustering data of mRNAs in gliomas were analyzed

of 4,858 (4330-5153) IncRNAs were differentially expressed in glioma compared to peritumoral tissue. These consisted of 2,845 (2,610-3,228) up-regulated (fold change > 3.0) IncRNAs and 2,013 (1,720-2,395) down-regulated (fold change $<1 / 3$ ) IncRNAs. In tissues obtained from the three glioma patients, there were 1,290 IncRNAs that were up-regulated or down-regulated more than 3-fold; additionally, 494 were changed (either up or down) more than 5-fold, and 145 IncRNAs altered by greater than 10 -fold. Of all differentially expressed IncRNAs, uc002ukq.2 (log2 fold change $\mathrm{T} / \mathrm{N}=8.50108)$ was the most significantly up-regulated and AK002107 (log2 fold change $\mathrm{T} / \mathrm{N}=$ $-10.64121)$ was the most significantly down-regulated.
Of all differentially expressed IncRNAs, a majority were up-regulated in glioma; in contrast, a smaller percentage of IncRNAs were down-regulated in tumor tissue (Figure 1 B). Differentially expressed IncRNAs and mRNAs are provided in Table II. In general, the greater the fold change value, the more likely that a particular IncRNA is involved in tumor development. To narrow down our list, we chose 6 IncRNAs (ak125809, ak098473, uc002ehu.1, bc043564, NR_027322, and uc003qmb.2) showing the greatest fold changes and the highest raw intensity values for further investigation.

\section{Aberrantly expressed mRNAs in glioma}

We identified 18,315 mRNA transcripts that were differentially expressed between tumor 
Table II. Summary of data from microarray for three pairs of glioma and adjacent normal tissues

\begin{tabular}{|c|c|c|c|c|c|c|}
\hline Relative long nonc & ding RNA expressi & on between thr & glioma and & adjacent norm & sues & \\
\hline Sample & & & & g non-coding & & \\
\hline ID & & $\begin{array}{c}\text { Fold change } \\
>3\end{array}$ & $\begin{array}{c}\text { Fold change } \\
>5\end{array}$ & $\begin{array}{l}\text { Fold change } \\
>10\end{array}$ & Total & $\begin{array}{c}\text { Changed } \\
\text { IncRNA }\end{array}$ \\
\hline Couple1 & Up-regulation & 2029 & 945 & 254 & 3228 & 5153 \\
\hline$(0301 \text { vs. } 0601)^{\star}$ & Down-regulation & 1051 & 567 & 307 & 1925 & \\
\hline Couple2 & Up-regulation & 1668 & 741 & 201 & 2610 & 4330 \\
\hline (0605 vs. 0601) & Down-regulation & 1084 & 403 & 233 & 1720 & \\
\hline Couple3 & Up-regulation & 1538 & 794 & 464 & 2696 & 5091 \\
\hline (114982 vs. 0601) & Down-regulation & 1330 & 680 & 385 & 2395 & \\
\hline Relative messenge & RNA expression b & tween three $\mathrm{g}$ & oma and adja & ent normal tis & & \\
\hline Sample & & & & Messenger RN & & \\
\hline & & $\begin{array}{c}\text { Fold change } \\
\quad>3\end{array}$ & $\begin{array}{c}\text { Fold change } \\
\quad>5\end{array}$ & $\begin{array}{l}\text { Fold change } \\
>10\end{array}$ & Total & $\begin{array}{c}\text { Changed } \\
\text { mRNA }\end{array}$ \\
\hline Couple1 & Up-regulation & 1363 & 673 & 244 & 2280 & 4143 \\
\hline (0301 vs. 0601) & Down-regulation & 964 & 445 & 454 & 1863 & \\
\hline Couple2 & Up-regulation & 1106 & 558 & 272 & 1936 & 3548 \\
\hline (0605 vs. 0601) & Down-regulation & 916 & 398 & 298 & 1612 & \\
\hline Couple3 & Up-regulation & 1353 & 856 & 417 & 2626 & 4564 \\
\hline (114982 vs. 0601) & Down-regulation & 903 & 536 & 499 & 1938 & \\
\hline
\end{tabular}

tissue and normal tissue. An average of 2,280 $(1,936-2,626)$ mRNAs were up-regulated more than 3 -fold in tumor tissue and an average of $1,804(1,612-1,938)$ mRNAs were down-regulated (fold change $<1 / 3$ ) in tumors. In the three glioma samples, 1,735 mRNAs were differentially expressed greater than 3-fold, 762 mRNAs greater than 5 -fold, and 272 mRNAs greater than 10-fold (Table II).

\section{Pathway analysis}

Pathway analysis was performed to identify the major signaling pathways activated in tumor cells. We identified 19 pathways that were associated with aberrantly up-regulated transcripts; the most highly enriched pathway was Cell cycle - Homo sapiens (human) consisting of 34 differentially expressed target genes. In contrast, 39 pathways were associated with down-regulated transcripts; the most highly enriched pathway in this group was Salivary secretion - Homo sapiens (human) consisting of 21 target genes (recommended $p$-value cut-off $=0.05)$. Based on these analyses, we can also conclude that the "GLIOMA" signaling pathway (KEGG Pathway ID: hsa05214) was clearly involved in glioma development (Figure 2).

\section{Establishing gene co-expression networks}

We established IncRNA-mRNA co-expression networks to further investigate the connection between these two types of molecules. Only IncRNAs and mRNAs that were at least 5-fold differentially expressed between tumor and normal tissue were selected for construction of the co-expression network (Figure 3). Co-expression networks were constructed based on the expression values of differentially expressed IncRNAs and mRNAs in each sample. Since gene expression in tumors reflected the regulatory relationships among genes, the co-expression networks of differentially expressed IncRNAs and mRNAs were constructed based on tumor-specific conditions.

The network property of each node was analyzed based on the co-expression network. The degree of each gene in the network was used to predict mRNA/ IncRNA interaction. A list of mRNAs and IncRNAs with the highest degrees is provided in Table III.

Using the mRNA/IncRNA network, we identified 119 IncRNAs and 335 mRNAs showing some degree of interaction in glioma (correlation $=0.99, p=$ 0.007). Of these, LOC729991 and NUDCD1 mRNAs had the highest degree value. ENST00000425922 and ENST00000455568 IncRNAs were the most 
A

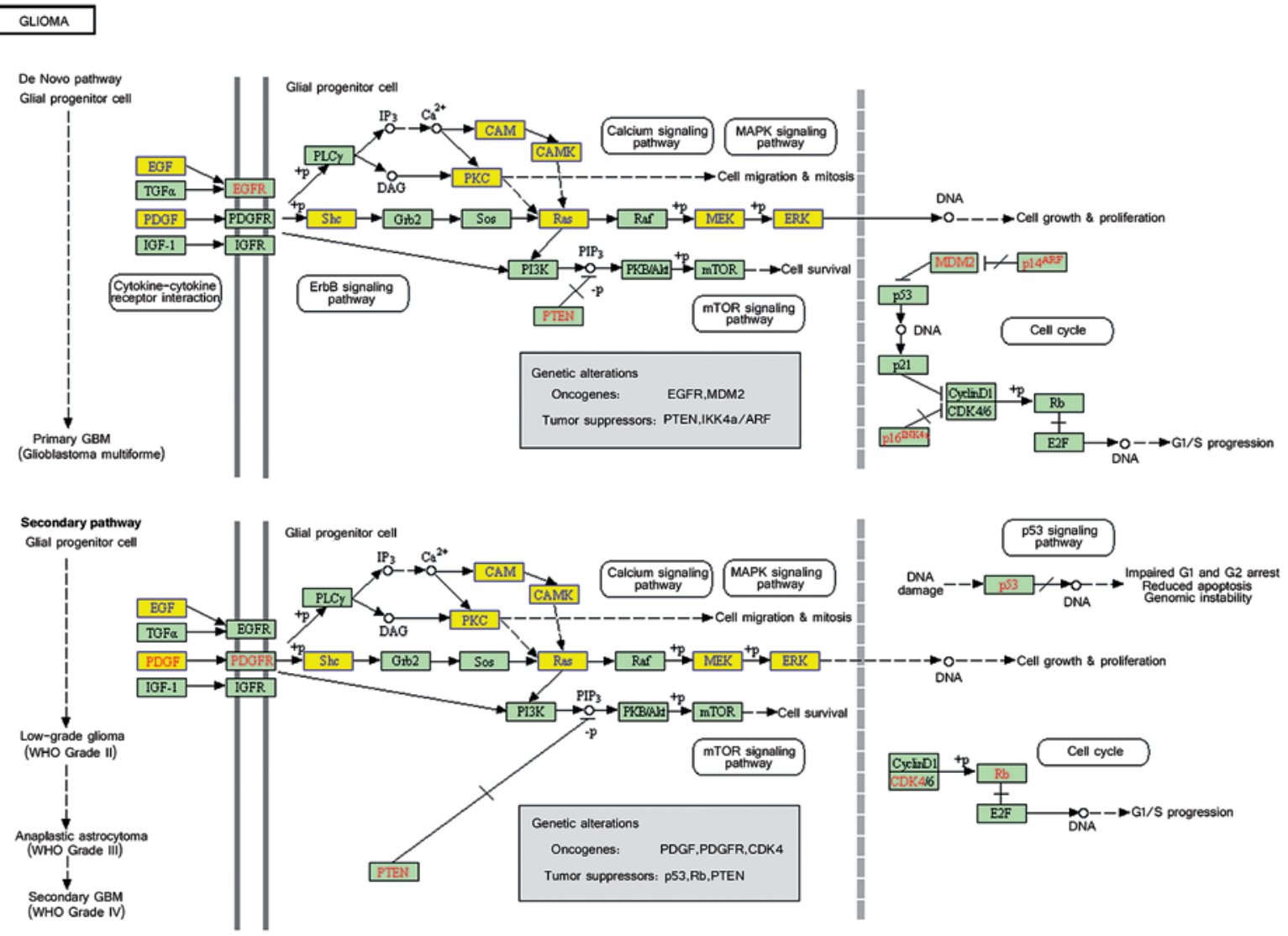

Figure 2. Pathway analysis of aberrantly expressed transcripts in gliomas. A - Key genes belonging to the "GLIOMA" signaling pathway (KEGG: hsa05214), including PDGF, SHC, MDM2, and EGFR, also exhibit close relationships with IncRNAs

extensively connected with other mRNAs and $\ln$ cRNAs in the network. Additionally, key genes of the "GLIOMA" signaling pathway, including SHC3, PDGFA, and MDM2, all had high degree scores.

\section{RT-qPCR verification}

IncRNAs that were differentially expressed in all three tumor groups and had fold changes $>10$ were arranged in descending order based on normalized intensity. The top six IncRNAs in this group (ak125809, ak098473, uc002ehu.1, bc043564, NR 027322, and uc003qmb.2) were validated by qPCR. We found that IncRNAs ak125809, ak098473, and uc002ehu.1 were up-regulated in over $90 \%$ of the 40 glioma cases. In contrast, IncRNAs bc043564, NR 027322, and uc003qmb.2 were down-regulated in tumor tissue compared to normal. These data are consistent with the microarray results $(p=0.03)$ (Figure 4$)$.

\section{Discussion}

The genetic basis of glioma development has been extensively studied, and the molecular path- ways that become deregulated in the disease have also been examined $[27,28]$. Aberrant expression of both mRNA transcripts and microRNAs have been shown to be involved in glioma development and progression [29, 30]. One study performed miRNA expression profiling and found that differential expression of miRNAs in malignant astrocytomas may influence tumor proliferation and response to chemotherapy [31]. While a role for both mRNAs and miRNAs in glioma has been well studied, a role for IncRNAs in the disease has yet to be established. Identification of IncRNAs that are important for disease progression could result in new options for glioma prognosis and treatment [16, 32].

In this study, we performed IncRNA expression profiling using microarray technology. We identified thousands of IncRNAs that were differentially expressed in human glioma samples compared to peritumoral normal tissue. We selected 6 IncRNAs (ak125809, ak098473, uc002ehu.1, bc043564, NR_027322, and uc003qmb.2) with fold changes greater than 10 and with relatively high normalized intensities for qPCR validation in 40 pairs of 


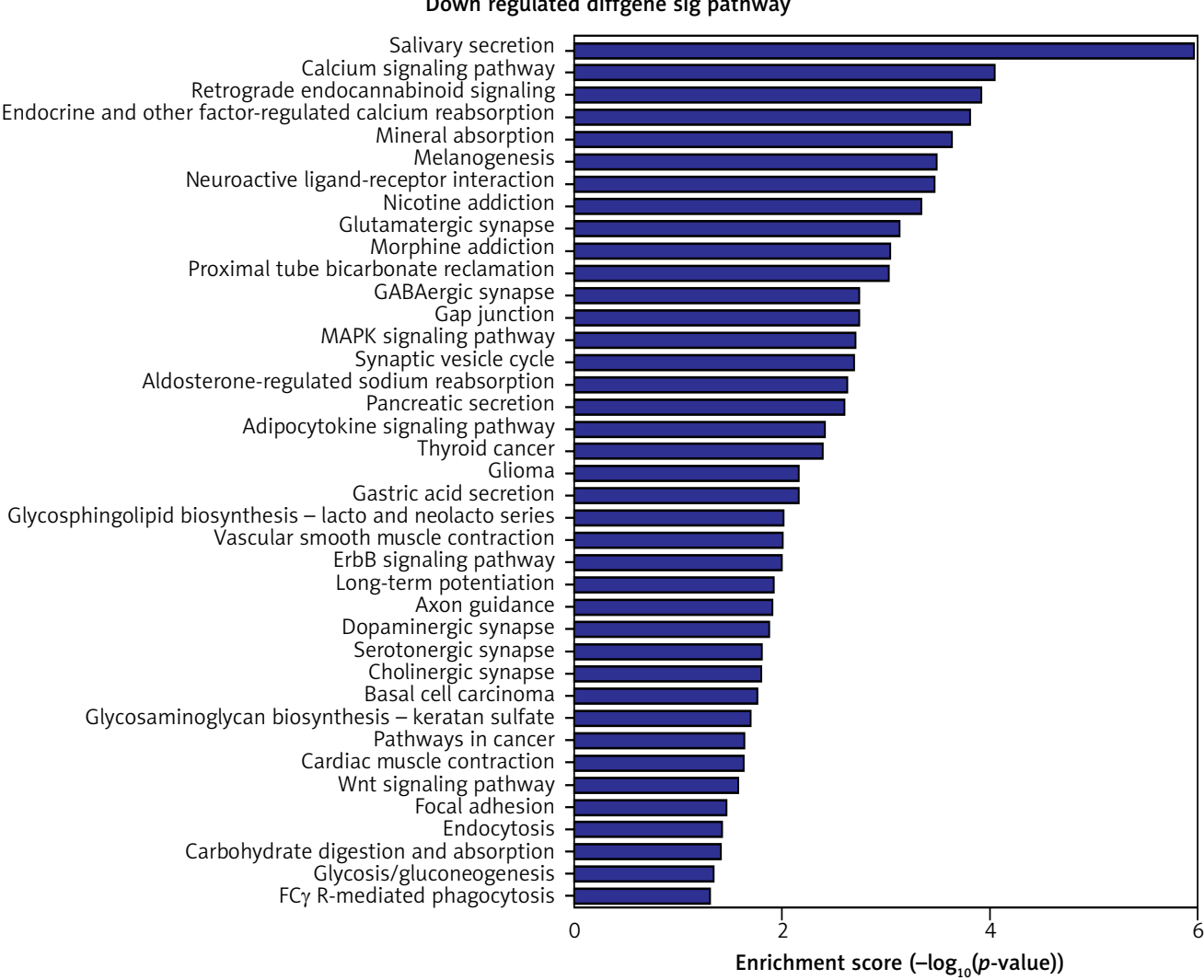

C

Up regulated diffgene sig pathway

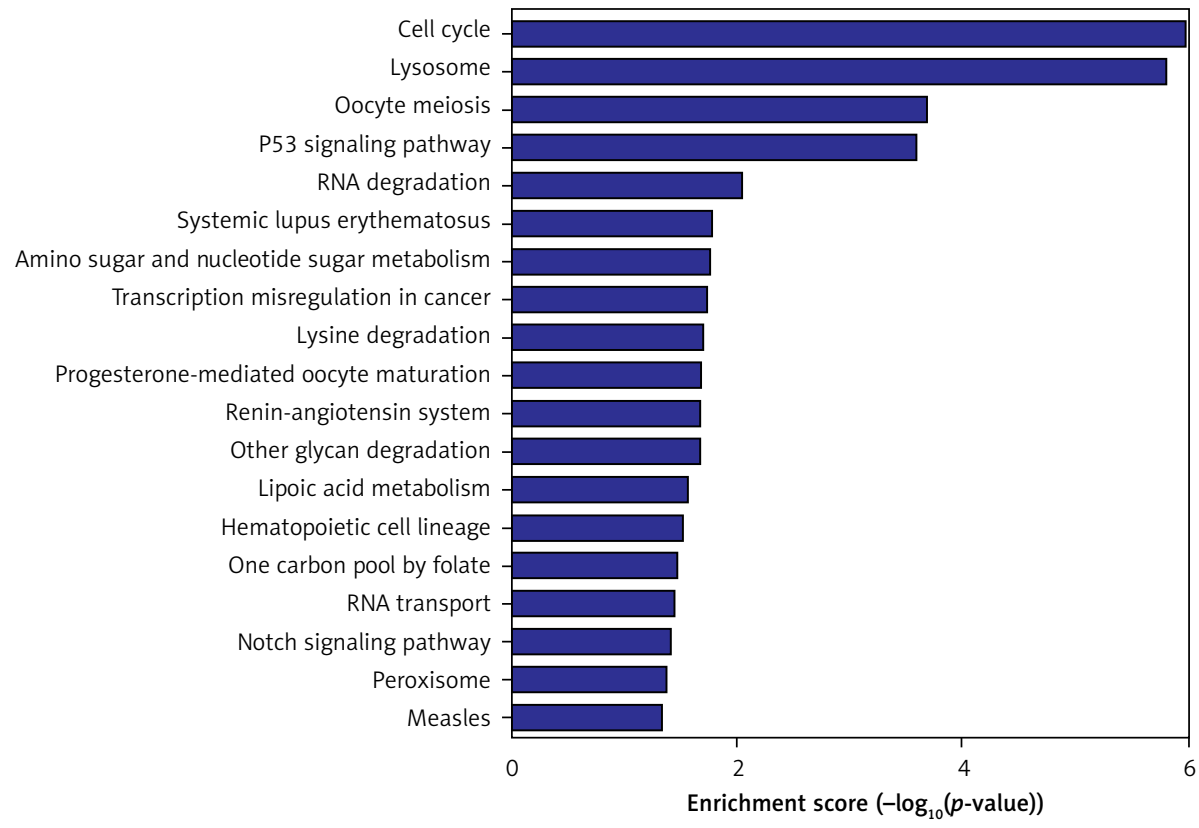

Figure 2. Cont. B - Pathway-associated down-regulated mRNAs are provided. C - Pathway-associated up-regulated mRNAs are provided

gliomas and their surrounding tissue. We hypothesize that these IncRNAs may be potential biomarkers in glioma. Additionally, the IncRNA HULC may be useful as a biomarker capable of being detected in plasma [12].

One of the 6 IncRNAs that was extensively examined in this study, ak125809, was upregulated in tumor tissue. ak125809 is located on chr7 and has a length of $3854 \mathrm{nt}$. It is the natural antisense transcript of the gene encoding vasoactive intestinal peptide receptor 2 (VIPR2), also known as PACAP-R-3. VIPR2-mediated signal transduction results in glial cell proliferation, a process that is closely related to glioma formation and 


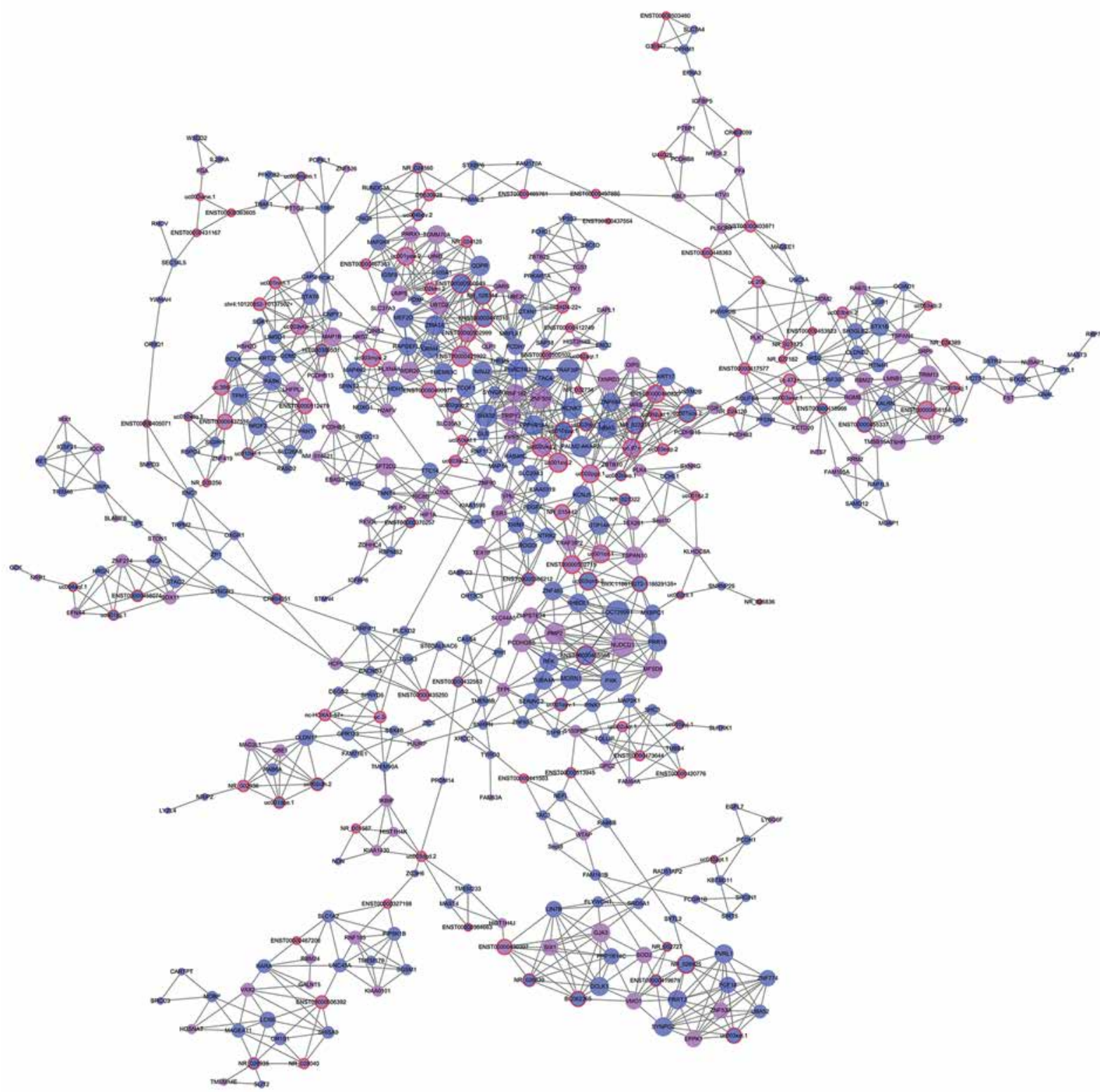

Figure 3. LncRNA-mRNA co-expression network: nodes with red cycle represent IncRNAs, nodes without cycle represent mRNAs, straight lines represent interactions between genes, purple represents increased expression, and blue represents decreased expression. The size of the node represents the degree; the higher the degree, the more genes interact with the particular node in the network

repair following neurological damage. Ak125809 may have an indirect relation to glioma proliferation due to its regulation of VIPR2 expression [33]. The IncRNA BC043564, located on chr1 with a length of $2022 \mathrm{nt}$, is the exon sense-overlapping transcript of the Kcn2a gene, which encodes one subtype of proliferation-related voltage-gated potassium ion channel [34]. The IncRNA ak098473, located on chrX with a length of 1658 $n t$, is the natural antisense transcript of the gene encoding synaptotagmin-like protein 4 (SYTL4). The IncRNA uc002ehu.1 is located on chr17 and has a length of 643 nt. NR_027322 is located on chr10 with a length of 2427 nt. The biological function of the latter three IncRNAs is currently unknown.

We identified several oncogene-related IncRNAs in our microarray analysis. BC007968 was found to be significantly upregulated; it is a natural antisense transcript of cyclin-dependent kinase 4 (CDK4), a Ser/Thr protein kinase. Expression of CDK4 and its associated proteins, including D-type cyclins, p16 (INK4a), and Rb, has been shown to be important for glioma tumorigenesis; moreover, disruption of the p16INK4a-Cdk4-Rb pathway has been shown to be useful for glioma therapy [35, 36]. BC007968 is very likely to interfere with the p16INK4a-Cdk4-Rb pathway as a CDK4 antisense 
Table III. Degree was used to assess interactions in the IncRNA/mRNA network. This table is a collection of a series of key IncRNA/mRNAs

\begin{tabular}{|c|c|c|c|c|c|c|c|}
\hline Gene symbol & Style & Regulation & Degree & $\begin{array}{c}\text { Gene } \\
\text { symbol }\end{array}$ & Style & Regulation & Degree \\
\hline ENST00000425922 & Up & LncRNA & 13 & LOC729991 & Down & mRNA & 15 \\
\hline ENST00000455568 & Down & LncRNA & 12 & NUDCD1 & Up & mRNA & 15 \\
\hline ENST00000500949 & Down & LncRNA & 12 & SNX32 & Down & mRNA & 14 \\
\hline uc001xvj.2 & Up & LncRNA & 12 & TRAF3IP1 & Down & mRNA & 14 \\
\hline uc002ukq.2 & Up & LncRNA & 12 & KCNK7 & Down & mRNA & 13 \\
\hline uc003qmb.2 & Down & LncRNA & 11 & MORN1 & Down & mRNA & 13 \\
\hline ENST00000502715 & Up & LncRNA & 11 & $\begin{array}{l}\text { PALM2- } \\
\text { AKAP2 }\end{array}$ & Down & mRNA & 13 \\
\hline ENST00000502999 & Up & LncRNA & 11 & PMP2 & Up & mRNA & 13 \\
\hline NR_028344 & Down & LncRNA & 11 & PXK & Down & mRNA & 13 \\
\hline uc002pgz.1 & Up & LncRNA & 11 & TXNRD3 & Up & mRNA & 13 \\
\hline ENST00000450820 & Up & LncRNA & 11 & ZNF501 & Up & mRNA & 13 \\
\hline
\end{tabular}

A

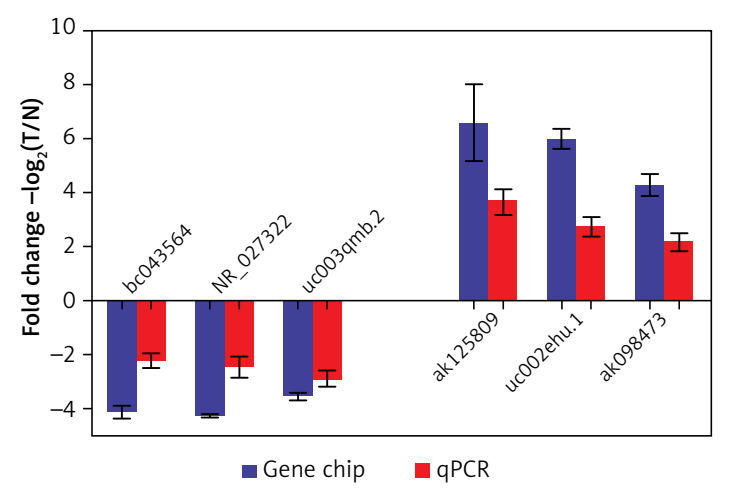

B

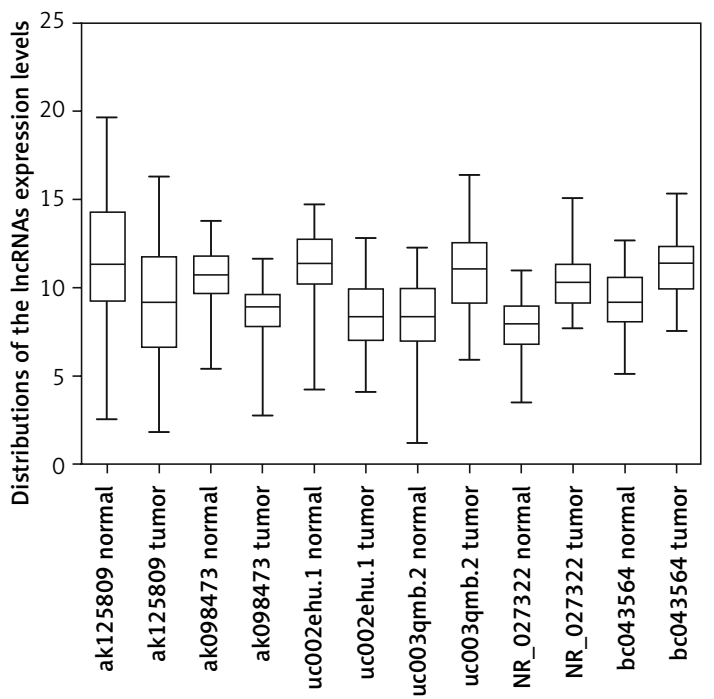

Figure 4. Comparison of microarray data and $q P C R$ results. A - qPCR was used to verify expression of IncRNAs ak125809, ak098473, uc002ehu.1, bc043564, NR_027322, and uc003qmb.2. B - Distribution of IncRNA expression levels were provided. All six IncRNAs of ak125809, ak098473, uc002ehu.1, bc043564, NR_027322, and uc003 mb. 2 were validated by qPCR analysis in the 40 paired glioma and peritumoral tissues. Each histogram represents the average fold change $(T / N)$ with logarithmic conversion. Error bars are indicative of standard deviation. Distribution of IncRNA expression

transcript and would be predicted to regulate glioma formation. The IncRNA BC008580 was also upregulated in glioma. This is an exon sense-overlapping transcript of glial cell line-derived neurotrophic factor (GDNF). Recent work has shown that GDNF is overexpressed in glioma, where it likely contributes to increased proliferation and metastasis [37, 38]. It is possible that the IncRNA BC008580 contributes to glioma development via regulation of GDNF. Taken together, our data support a role for IncRNAs in glioma tumorigenesis.
Interaction or co-expression networks can be generated based on multiple assays, including co-immunoprecipitation experiments [39] and gene function analyses [40]. We generated an IncRNA-mRNA expression network based on data from our microarray expression profiling. We determined Pearson correlation coefficients and selected highly correlated IncRNA-mRNA pairs to establish the network. The network indicated which IncRNAs might be responsible for regulation of specific mRNAs. While mRNAs seemed to directly 
influence tumorigenesis and disease malignancy, IncRNAs tended to have a more indirect effect, possibly via regulation of downstream mRNAs. Degree scores were assigned to molecules in the network, and those with the highest degree scores likely displayed the greatest extent of interaction. Through these analyses, we identified potential IncRNA-mRNA interactions. A better understanding of these interactions will likely help elucidate some key roles for IncRNAs in glioma.

We performed KEGG annotation of all mRNAs differentially expressed between glioma and normal tissue. Several pathways, including MAPK signaling, the Wnt pathway, p53 signaling, and the glioma pathway, were all found to be deregulated in the disease. Most of these pathways have been previously shown to influence glioma tumorigenesis and response to chemotherapy. For example, one study showed that down-regulation of Wnt2 and beta-catenin inhibited proliferation of malignant glioma [41]. Additionally, work by Johannessen et al. showed that activation of p53 by the MDM2 antagonist nutlin-3 improved sensitivity to chemotherapy treatment [42]. Of all pathways identified by KEGG analysis, the "Glioma" pathway (KEGG Pathway ID: hsa05214) particularly stood out [43-45]. Several genes belonging to this pathway, including PDGF, SHC, MDM2, and EGFR, have previously been shown to be important for glioma development [46, 47]. Many of these genes were also found to be differentially expressed in this study. Additionally, our IncRNA-mRNA co-expression network analysis showed that several of these genes correlate with particular IncRNAs. For example, shc3 correlated with uc002ukz.1, enst00000473644, and uc010ayj.1 (correlation = 1, $p<0.01$ ). Pdgfa correlated with enst00000502715 and NR_022011.Mdm2 correlated with NR_027873 and uc.208- (correlation $=1, p<0.01$ ). We hypothesize that many of these IncRNAs regulate glioma formation by interfering with the expression of key factors in the "Glioma" pathway. We plan to test this hypothesis in future studies.

In this study, we identified differentially expressed IncRNAs in human glioma cases by both microarray profiling and QPCR validation. Importantly, data from both microarray and qPCR were consistent. We established an IncRNA-mRNA co-expression network to better understand the mechanisms of IncRNA action in the disease. We found IncRNA that may be associated with the pathogenesis of glioma, as a phenomenon, but the mechanism was not further explored, and whether it can be detected in blood and body fluids and become a biomarker is yet to be studied. The project team will further study this series of issues later. While this work provides a solid foundation, more research must be done to clearly define the molecular roles of IncRNAs in glioma development.

\section{Acknowledgments}

This work was supported by the Starting Fund for Young Scientists of Nanjing Public Health Bureau (QYK11126).

Yi Ding, Xinfa Wang and Junchen Pan have equal contuibute to this paper. Thanks to Mingjun Jis laboratory and their colleagues.

\section{Conflict of interest}

The authors declare no conflict of interest.

\section{References}

1. Patnaik A, Mishra SS, Senapati SB. Intraventricular glioblastoma multiforme mimicking meningioma and review of the literature. Asian J Neurosurg 2017; 12: 75-7.

2. Ohgaki H, Kleihues P. Epidemiology and etiology of gliomas. Acta Neuropathol 2005; 109: 93-108.

3. Zhong D, Ran J, Zhang X, et al. Syntenin is expressed in human gliomas and may correlate with tumor migration. Arch Med Sci 2015; 11: 1303-7.

4. Wilusz JE, Sunwoo H, Spector DL. Long noncoding RNAs: functional surprises from the RNA world. Genes Dev 2009; 23: 1494-504.

5. Zhao J, Sun BK, Erwin JA, Song JJ, Lee JT. Polycomb proteins targeted by a short repeat RNA to the mouse $\mathrm{X}$ chromosome. Science 2008; 322: 750-6.

6. Pandey RR, Mondal T, Mohammad F, et al. Kenq1ot1 antisense noncoding RNA mediates lineage-specific transcriptional silencing through chromatin-level regulation. Mol Cell 2008; 32: 232-46.

7. Wang X, Arai S, Song X, et al. Induced ncRNAs allosterically modify RNA-binding proteins in cis to inhibit transcription. Nature 2008; 454: 126-30.

8. Beltran M, Puig I, Peña C, et al. A natural antisense transcript regulates Zeb2/Sip1 gene expression during Snail1-induced epithelial-mesenchymal transition. Genes Dev 2008; 22: 756-69.

9. Yu G, Yao W, Wang J, et al. LncRNAs expression signatures of renal clear cell carcinoma revealed by microarray. PLoS One 2012; 7: e42377.

10. Xu C, Yang M, Tian J, Wang X, Li Z. MALAT-1: a long non-coding RNA and its important 3' end functional motif in colorectal cancer metastasis. Int J Oncol 2011; 39: 169-75.

11. Birney E, Stamatoyannopoulos JA, Dutta A, et al. Identification and analysis of functional elements in $1 \%$ of the human genome by the ENCODE pilot project. Nature 2007; 447: 799-816.

12. Panzitt K, Tschernatsch MM, Guelly C, et al. Characterization of HULC, a novel gene with striking up-regulation in hepatocellular carcinoma, as noncoding RNA. Gastroenterology 2007; 132: 330-42.

13. Yuan SX, Yang F, Yang Y, et al. Long noncoding RNA associated with microvascular invasion in hepatocellular carcinoma promotes angiogenesis and serves as a predictor for hepatocellular carcinoma patients' poor recurrence-free survival after hepatectomy. Hepatology 2012; 56: 2231-41.

14. Bracken AP, Helin K. Polycomb group proteins: navigators of lineage pathways led astray in cancer. Nat Rev Cancer 2009; 9: 773-84. 
15. Calin GA, Liu CG, Ferracin $M$, et al. Ultraconserved regions encoding ncRNAs are altered in human leukemias and carcinomas. Cancer Cell 2007; 12: 215-29.

16. Wang $P$, Ren Z, Sun P. Overexpression of the long non-coding RNA MEG3 impairs in vitro glioma cell proliferation. J Cell Biochem 2012; 113: 1868-74.

17. Benetatos L, Vartholomatos G, Hatzimichael E. MEG3 imprinted gene contribution in tumorigenesis. Int J Cancer 2011; 129: 773-9.

18. Han L, Zhang K, Shi Z, et al. LncRNA profile of glioblastoma reveals the potential role of IncRNAs in contributing to glioblastoma pathogenesis. Int J Oncol 2012; 40: 2004-12.

19. Zhang X, Sun S, Pu JK, et al. Long non-coding RNA expression profiles predict clinical phenotypes in glioma. Neurobiol Dis 2012; 48: 1-8.

20. Guo Y, Vickers K, Xiong Y, et al. Comprehensive evaluation of extracellular small RNA isolation methods from serum in high throughput sequencing. BMC Genomics 2017; 18: 50.

21. Han B, Bu P, Meng X, Hou X. Microarray profiling of long non-coding RNAs associated with idiopathic pulmonary arterial hypertension. Exp Ther Med 2017; 13: 2657-66.

22. Shang D, Zheng T, Zhang J, Tian Y, Liu Y. Profiling of mRNA and long non-coding RNA of urothelial cancer in recipients after renal transplantation. Tumour Biol 2016; 37: 12673-84

23. Pfaffl MW. A new mathematical model for relative quantification in real-time RT-PCR. Nucleic Acids Res 2001; 29: e45.

24. Draghici S, Khatri P, Tarca AL, et al. A systems biology approach for pathway level analysis. Genome Res 2007; 17: 1537-45

25. Yi M, Horton JD, Cohen JC, Hobbs HH, Stephens RM WholePathwayScope: a comprehensive pathway-based analysis tool for high-throughput data. BMC Bioinformatics 2006; 7: 30.

26. Kanehisa M, Goto S, Kawashima S, Okuno Y, Hattori M. The KEGG resource for deciphering the genome. Nucleic Acids Res 2004; 32: D277-80.

27. Yang H, Zhang QX, Pei DS, Xu F, Li Y, Yu RT. FK506-binding protein 5 inhibits proliferation and stimulates apoptosis of glioma cells. Arch Med Sci 2015; 11: 1074-80.

28. Hong Y, Shi Y, Shang C, Xue Y, Liu Y. Influence of far upstream element binding protein 1 gene on chemotherapy sensitivity in human U251 glioblastoma cells. Arch Med Sci 2016; 12: 156-62

29. Vitucci M, Hayes DN, Miller CR. Gene expression profiling of gliomas: merging genomic and histopathological classification for personalised therapy. Br J Cancer 2011; 104: 545-53.

30. Shai R, Shi T, Kremen TJ, et al. Gene expression profiling identifies molecular subtypes of gliomas. Oncogene 2003; 22: 4918-23.

31. Rao SA, Santosh V, Somasundaram K. Genome-wide expression profiling identifies deregulated miRNAs in malignant astrocytoma. Mod Pathol 2010; 23: 1404-17.

32. Barsyte-Lovejoy D, Lau SK, Boutros PC, et al. The c-Myc oncogene directly induces the H19 noncoding RNA by allele-specific binding to potentiate tumorigenesis. Cancer Res 2006; 66: 5330-7.

33. Jaworski DM. Expression of pituitary adenylate cyclase-activating polypeptide (PACAP) and the PACAP-selective receptor in cultured rat astrocytes, human brain tumors, and in response to acute intracranial injury. Cell Tissue Res 2000; 300: 219-30.
34. Akhtar S, McIntosh P, Bryan-Sisneros A, Barratt L, Robertson B, Dolly JO. A functional spliced-variant of beta 2 subunit of Kv1 channels in $\mathrm{C} 6$ glioma cells and reactive astrocytes from rat lesioned cerebellum. Biochemistry 1999; 38: 16984-92.

35. Parsons DW, Jones S, Zhang X, et al. An integrated genomic analysis of human glioblastoma multiforme. Science 2008; 321: 1807-12.

36. Cancer Genome Atlas Research Network: Comprehensive genomic characterization defines human glioblas toma genes and core pathways. Nature 2008; 455 : 1061-8.

37. Ng WH, Wan GQ, Peng ZN, Too HP. Glial cell-line derived neurotrophic factor (GDNF) family of ligands confer chemoresistance in a ligand-specific fashion in malignant gliomas. J Clin Neurosci 2009; 16: 427-36.

38. Golan M, Schreiber G, Avissar S. Antidepressants elevate GDNF expression and release from $C(6)$ glioma cells in a beta-arrestin1-dependent, CREB interactive pathway. Int J Neuropsychopharmacol 2011; 14: 1289-300.

39. Smidtas S, Yartseva A, Schachter V, Kepes F. Model of interactions in biology and application to heterogeneous network in yeast. C R Biol 2006; 329: 945-52.

40. Nikiforova VJ, Willmitzer L. Network visualization and network analysis. EXS 2007; 97: 245-75.

41. Pu P, Zhang Z, Kang C, et al. Downregulation of Wnt2 and beta-catenin by siRNA suppresses malignant glioma cell growth. Cancer Gene Ther 2009; 16: 351-61.

42. Johannessen TC, Prestegarden L, Grudic A, Hegi ME, Tysnes BB, Bjerkvig R. The DNA repair protein ALKBH2 mediates temozolomide resistance in human glioblastoma cells. Neuro Oncol 2013; 15: 269-78.

43. Bansal K, Liang ML, Rutka JT. Molecular biology of human gliomas. Technol Cancer Res Treat 2006; 5: 185-94

44. Kapoor GS, O’Rourke DM. Mitogenic signaling cascades in glial tumors. Neurosurgery 2003; 52: 1425-34; discussion 1434-25.

45. Hulleman E, Helin K. Molecular mechanisms in gliomagenesis. Adv Cancer Res 2005; 94: 1-27.

46. Martinho O, Longatto-Filho A, Lambros MB, et al. Expression, mutation and copy number analysis of platelet-derived growth factor receptor A (PDGFRA) and its ligand PDGFA in gliomas. Br J Cancer 2009; 101: 973-82.

47. Ortensi B, Osti D, Pellegatta S, et al. Rai is a new regulator of neural progenitor migration and glioblastoma invasion. Stem Cells 2012; 30: 817-32. 